\title{
Insegurança Alimentar em Região de Alta Vulnerabilidade Social da Cidade de São Paulo
}

\author{
Daniela de Almeida Pereira ${ }^{1}$, Viviane Laudelino Vieira ${ }^{2}$, Elaine Gomes Fiore ${ }^{3}$ e Ana Maria Cervato-Mancuso ${ }^{1}$
}

Com o objetivo de descrever algumas características das famílias acompanhadas pela Pastoral da Criança em região de alta vulnerabilidade social da cidade de São Paulo, com relação aos aspectos sócio-demográficos e identificação da situação de segurança alimentar e nutricional, utilizou-se, respectivamente, um questionário e um instrumento validado para classificação familiar segundo a Escala Brasileira de Insegurança Alimentar (EBIA). Verificou-se que a maioria das famílias era composta por até 6 pessoas, sendo comum a presença de no máximo três crianças menores de seis anos. O recebimento de doação na forma de alimentos foi relatado por mais da metade das famílias. Declararam apresentar geração de renda (trabalho registrado ou não) 62 (75\%) famílias, sendo que $73(95,1 \%)$ apresentavam renda per capita inferior a 0,5 salário mínimo. A situação de segurança alimentar foi observada em apenas $12 \%$ das famílias. Conclui-se que a situação de insegurança alimentar familiar predomina na população estudada, bem como renda familiar insuficiente e expressiva taxa de desemprego, fatores atrelados à condição de alta vulnerabilidade social.

Palavras-chave: segurança alimentar, vulnerabilidade social, população urbana.

\section{Food Insecurity in a Zone of High Social Vulnerability of the City of São Paulo}

\begin{abstract}
With the objective of describing some characteristics of the families assisted by the Pastoral da Crianca in a region of high social vulnerability of the city of São Paulo, with regard to the socio-demographic aspects and the identification of the food security status, for which a questionnaire and an instrument for family classification validated according to the Escala Brasileira de Insegurança Alimentar (EBIA). We found that the majority of families were made up of up to six individuals, each having up to three children of six years of age or under. More than the half of the families declared receiving food donations. Sixty-two (75\%) families declared to income generating (whether legally registered or not) while 73 (95.1\%) earned a per-capita income below 0.5 minimum wage. A situation of food security was observed in only $12 \%$ of the families. It is concluded that family food insecurity predominates in the studied population, as are an insufficient family income and high unemployment; factors closely associated to the condition of high social vulnerability.
\end{abstract}

Keywords: food security, social vulnerability, urban population

\footnotetext{
${ }^{1}$ Faculdade de Saúde Pública (USP)

${ }^{2}$ Centro de Saúde Escola Geraldo de Paula Souza da Faculdade de Saúde Pública (USP)

${ }^{3}$ Universidade de Guarulhos
} 


\section{Introdução}

O conceito brasileiro de segurança alimentar e nutricional consiste na realização do direito de todos ao acesso regular e permanente a alimentos de qualidade, em quantidade suficiente, sem comprometer o acesso a outras necessidades essenciais e tendo como base práticas alimentares promotoras de saúde, que respeitem a diversidade cultural e que sejam social, econômica e ambientalmente sustentáveis ${ }^{[1,2]}$.

Assim, quando ocorre a violação do direito humano à alimentação e não é assegurado o acesso permanente a alimentos de qualidade e em quantidade suficiente ou às condições para sua obtenção, são geradas situações de insegurança alimentar ${ }^{[3]}$.

A insegurança alimentar relaciona-se com a vulnerabilidade social por resultar de uma combinação de fatores que podem produzir uma deterioração do nível de bem-estar de pessoas, famílias ou comunidades, conforme a exposição a determinados tipos de riscos ${ }^{[4]}$.

A insuficiência de renda, o elevado nível de desemprego, a concentração de terra, a mercantilização da água e a precarização da educação limitam o acesso à alimentação e nutrição e impedem uma vida digna para toda população brasileira ${ }^{[1]}$.

O surgimento de problemas que interferem na oferta, no acesso físico e econômico, no consumo ou no padrão de utilização biológica dos alimentos, ou seja, que resultem em situação de insegurança alimentar, pode levar ao desenvolvimento de doenças e agravos endêmicos, como a desnutrição, as carências específicas, a obesidade, o diabetes melito, as dislipidemias e as associações com outras doenças crônicas de reconhecida relevância epidemiológica e cuja resolução compete ao setor de Saúde ${ }^{[3]}$.

Considerando-se ainda que a segurança alimentar e nutricional integra um conjunto de direitos que definem a qualidade de vida e pressupõe o fim da exclusão social, é papel do governo municipal, por meio do diagnóstico da insegurança alimentar, definir políticas públicas que visem garantir o direito à alimentação e a redução da exclusão social $^{[5]}$.

A Subprefeitura do Butantã, na zona oeste da cidade de São Paulo, reúne cerca de 377 mil habitantes, dos quais 30 mil vivem em favelas situadas em terrenos próximos a córregos e áreas que, no período das chuvas, transformam-se em regiões alagadas, difundindo doenças pela aguda deficiência na captação e tratamento de esgotos, que muitas vezes correm a céu aberto. São identificados pela subprefeitura do Butantã nove bolsões de pobreza na região, sendo o Jardim Jaqueline, com $10 \mathrm{mil}$ habitantes, o maior e o mais crítico dos bolsões, tanto em termos de habitat, quanto à pobreza de seus habitantes, que resulta em altíssimo índice de privação e vulnerabilidade social ${ }^{[6]}$.

Desde 2002, o Jardim Jaqueline é sede de um projeto do Instituto Polis para criação de um Centro de Referência em Segurança Alimentar e Nutricional (CRSAN), que contou com a parceria de técnicos da subprefeitura do Butantã, entidades sociais locais, equipamentos públicos e universidades, totalizando, atualmente, vinte e quatro entidades participantes do CRSAN ${ }^{[7]}$.

Dentre as entidades participantes do CRSAN, conta-se com a Pastoral da Criança, que trabalha nas áreas da saúde, nutrição e educação, desde o período gestacional até os seis anos de vida, e de prevenção da violência no ambiente familiar, envolvendo, necessariamente, as famílias e comunidades ${ }^{[8]}$. No Jardim Jaqueline, a ação da Pastoral da Criança desenvolve-se em três paróquias e conta com a participação de onze líderes para a realização das ações promovidas pela entidade.

A avaliação nutricional das crianças atendidas pela Pastoral do Jardim Jaqueline, em 2005, revelava que entre as crianças com, no máximo, dois anos, $6,7 \%$ apresentavam excesso de peso e, com proporção expressivamente inferior, 2,2\% apresentavam déficit de peso. Porém, entre as crianças maiores de dois anos 7,6\% apresentavam comprometimento de estatura e $1,9 \%$ excesso de peso ${ }^{[9]}$.

Este trabalho tem por objetivo descrever 
algumas características das famílias acompanhadas pelos agentes da Pastoral da Criança, com relação aos aspectos sócio-demográficos, incluindo a identificação da situação de segurança alimentar e nutricional.

\section{Metodologia}

Buscando respeitar os interesses dos sujeitos do estudo em sua integridade e dignidade, bem como o desenvolvimento de um trabalho dentro dos padrões éticos, o projeto em questão foi encaminhado ao Comitê de Ética em Pesquisa (CEP) da Faculdade de Saúde Pública da Universidade de São Paulo e, somente após sua aprovação pelo CEP, deu-se prosseguimento ao estudo.

Durante as atividades de pesagem das crianças, as famílias foram abordadas e convidadas a participar do estudo. Tal abordagem ocorreu de abril a junho de 2005, contabilizando a presença de 118 famílias, das quais 83 consentiram participar. As recusas ocorridas foram justificadas pela falta de disponibilidade de tempo das famílias para responder às questões (29 famílias) e pelo não interesse em participar (4 famílias). Foram excluídas duas famílias pelo fato do responsável pela criança residir no mesmo domicílio de outra família cadastrada anteriormente e pelo preenchimento incompleto dos questionários.

Os responsáveis pelo grupo familiar, que concordaram em participar do estudo, respondiam questões sobre a composição familiar (número de componentes, sexo, idade e escolaridade), histórico, renda, participação em programas de recebimento de auxílio ou alimentação e um questionário validado para avaliar a insegurança alimentar de famílias brasileiras, proposto por Segall-Correa et al ${ }^{[10]}$. Tal questionário é uma adaptação para a realidade brasileira de um módulo proposto pelo Departamento de Agricultura dos Estados Unidos (USDA), para avaliar a Insegurança Alimentar Domiciliar ${ }^{[1]}$.

O instrumento validado consiste em questões de múltiplas alternativas, das quais 15 são questões centrais fechadas (resposta sim ou não), seguidas de uma pergunta sobre a freqüência com que o evento afirmativo ocorre. As questões fechadas abordam a experiência de insuficiência alimentar nos últimos três meses, em seus diversos níveis de intensidade, desde a preocupação de que a comida possa vir a faltar até a vivência de passar todo um dia sem comer, permitindo classificar a família respondente na Escala Brasileira de Insegurança Alimentar - EBIA ${ }^{[12]}$.

Segundo a EBIA, para cada resposta às 15 questões levantadas atribui-se uma pontuação (1 ponto para resposta sim, 0 ponto para resposta não e não sabe). A somatória destes pontos permite a categorização familiar em segurança alimentar $(0$ pontos), insegurança leve (1 a 5 pontos), insegurança moderada (6 a 10 pontos) e insegurança severa (11 a 15 pontos), para domicílios com crianças e adolescentes ${ }^{[11]}$. As questões sobre a freqüência em que ocorre cada evento mencionado nas questões centrais, segundo a EBIA, não apresentam peso sobre a categorização familiar, não sendo por isso utilizadas no presente estudo.

Todos os dados relativos à caracterização das famílias e sobre a situação de insegurança alimentar familiar foram tabulados, utilizando-se o pacote estatístico EPIINFO versão $6.04{ }^{[13]}$. Adotou-se para a análise descritiva dos dados os valores de média e freqüência das variáveis selecionadas.

\section{Resultados}

Verificou-se que 59 (71,1\%) famílias eram compostas por até seis pessoas e 77 (85,5\%) famílias possuíam até 3 crianças com idade inferior a 6 anos. O número médio de filhos por domićlio era de 2,8, variando entre 1 e 10 filhos por família.

Em apenas 2 (2,4\%) famílias não havia mãe presente no domicílio, sendo uma por abandono do lar e outra por óbito. A média da idade materna era 29,6 anos, sendo que em $65(80,2 \%)$ famílias a idade materna encontrava-se entre 20 e 39 anos e em 5 $(6,2 \%)$ famílias a mãe apresentava idade inferior a 20 anos.

$\mathrm{Na}$ população estudada, foram identificadas $7(8,4 \%)$ gestantes e $26(31 \%)$ mulheres que amamentavam seus filhos, na época da coleta dos 
dados, com idade inferior a 6 meses. A escolaridade materna era representada pelo analfabetismo em 2 $(2,4 \%)$ famílias, pelo ensino fundamental incompleto em $50(60,2 \%)$ famílias e pelo ensino médio completo em $6(7,5 \%)$ famílias.

A presença de idoso foi relatada em apenas 3 (3,61\%) famílias, sendo o mesmo indicado como o chefe do domicílio e o membro que contribui com parte expressiva da renda total da família em que se encontra.

Cinquenta $(60,2 \%)$ famílias relataram ter nascido e sempre morado na cidade de São Paulo, sendo as demais provenientes da região sul $(1,3 \%)$, da região nordeste $(33,7 \%)$ e de outros municípios da região sudeste do país $(4,8 \%)$.

Mais da metade das famílias (53\%) recebe algum auxílio ou doação, como alimento (32,6\%), dinheiro $(12,3 \%)$ ou ambos $(8,1 \%)$. A maioria $(86,4 \%)$ das famílias informou que o auxílio recebido é utilizado por todos os seus membros. Quanto à origem do auxílio recebido, foram relatados programas do Governo Federal (43,2\%), como "Programa Bolsa Família", Entidades Religiosas (25\%), como "Pastoral da Criança", ou Entidades não-religiosas $(4,5 \%)$, como "Casa da Criança e do Adolescente Betinho".

O indivíduo indicado como chefe da família era do gênero masculino em 62 (74,6\%) famílias, podendo ser apontado como chefe tanto quem contribuía de forma mais significativa para a renda familiar, quanto quem era o responsável pelas decisões do núcleo familiar.

Tanto entre os homens como entre as mulheres, a escolaridade mais freqüente foi a de nível 1, ou seja, sem instrução, ensino fundamental completo ou incompleto.

Relataram possuir alguma fonte de geração de renda (com registro em carteira profissional ou não) 62 (75\%) famílias. Considerando-se a renda per capita mensal, $73(95,1 \%)$ famílias declararam receber menos que 0,5 salário mínimo* (Tabela 2).
Tabela 1: Distribuição percentual dos chefes de família segundo escolaridade e gênero. Jardim Jaqueline, São Paulo, 2005.

\begin{tabular}{ccccc}
\hline \multirow{2}{*}{ Escolaridade } & \multicolumn{2}{c}{ Feminino } & \multicolumn{2}{c}{ Masculino } \\
\cline { 2 - 5 } & No & $\mathbf{0}$ & No & $\mathbf{\%}$ \\
\hline Nível 1 & 12 & 70,58 & 48 & 81,36 \\
Nível 2 & 5 & 29,42 & 11 & 18,64 \\
\hline Total & 17 & 100,00 & 59 & 100,00 \\
\hline
\end{tabular}

Nível 1: Sem instrução, ensino fundamental incompleto e ensino fundamental completo.

Nível 2: Ensino médio incompleto, ensino médio completo e ensino superior inompleto

Tabela 2: Distribuição percentual das famílias segundo renda per capita mensal. Jardim Jaqueline, São Paulo, 2005.

\begin{tabular}{ccc}
\hline Renda per capita (SM) / mês & No & $\mathbf{\%}$ \\
\hline $0 \mid--0,25$ & 39 & 48,2 \\
$0,25 \mid--0,5$ & 28 & 34,6 \\
$0,5 \mid--1,0$ & 10 & 12,4 \\
$1 \mid--1,5$ & 4 & 4,9 \\
\hline TMtal & 81 & 100,0 \\
\hline = salário mínimo & &
\end{tabular}

Conforme sistema de pontuação das questões fechadas, 73 (88,0\%) famílias encontravam-se em situação de insegurança alimentar, sendo que somente $10(12,0 \%)$ famílias foram classificadas na situação de segurança alimentar (tabela 3).

Tabela 3: Distribuição das famílias segundo a EBIA. Jardim Jaqueline, São Paulo, 2005.

\begin{tabular}{ccc}
\hline Classificação & No & $\mathbf{0}$ \\
\hline Segurança alimentar & 10 & 12,0 \\
Insegurança alimentar leve & 37 & 44,6 \\
Insegurança alimentar moderada & 23 & 27,7 \\
Insegurança alimentar grave & 13 & 15,7 \\
\hline Total & 83 & 100,0 \\
\hline
\end{tabular}

* O valor do salário mínimo, entre abril e junho de 2005 (época da coleta de dados), era de $\mathrm{R} \$ 300,00$. 


\section{Discussão}

A constatação de famílias numerosas, com predomínio da presença de crianças, adolescentes e adultos jovens, combinada com a participação destacada de chefes de família jovens, apresentando ainda reduzidos rendimentos familiares $\mathrm{e}$ escolaridade, mostrou-se de acordo com a vulnerabilidade social da população estudada, a qual é classificada, conforme o Índice Paulista de Vulnerabilidade Social (IPVS) ${ }^{[4]}$, em condição de muito alta vulnerabilidade social.

Segundo Hoffmann ${ }^{[14]}$, a situação de insegurança alimentar é afetada pela presença ou não de pessoas com menos de 18 anos e pelo número de pessoas por domicílio. Assim, quanto maior o número de pessoas menores de 18 anos ou quanto maior o número de pessoas do domicílio, maior a situação de insegurança alimentar.

Conforme o IBGE ${ }^{[15]}$, que considera renda per capita mensal inferior a 0,5 SM mensal como parâmetro para definição da linha de pobreza, a população estudada apresenta $67(82,7 \%)$ famílias com seus membros classificados em tal condição, o que não causa estranheza levando-se em conta fatores como a insuficiência de renda, a precarização da educação e elevado número de membros por família em regiões de alta e muito alta vulnerabilidade social.

Deve-se destacar que a renda familiar é um fator relevante na situação de segurança/insegurança alimentar de uma população, uma vez que foi considerada pelo Grupo de Trabalho de Indicadores de Segurança Alimentar do Programa Comunidade Solidária (1998) como um dos sete indicadores-chave de insegurança alimentar ${ }^{[3]}$.

Hoffmann ${ }^{[16]}$ verificou, por meio da análise dos dados da pesquisa sobre segurança alimentar implementada pelo IBGE, em 2004, que a baixa renda domiciliar per capita é o determinante mais importante da insegurança alimentar, o que mostra a importância de programas de transferência de renda bem focalizados como o Bolsa-Família.

No entanto, seria muito limitado afirmar que a situação de insegurança alimentar é causada apenas pela insuficiência de renda familiar para comprar alimentos. Fatores como desemprego, produção de alimentos e direitos assegurados são também importantes para explicar a fome e, por conseguinte, a situação de segurança ou insegurança alimentar familiar ${ }^{[14]}$.

Considerando-se a questão da migração populacional, como o previsto em regiões de alta vulnerabilidade social, a maioria da população migrante é oriunda da região nordeste, provavelmente almejando melhores condições de vida na região sudeste ${ }^{[4]}$.

É importante destacar ainda que, de acordo com Hoffmann ${ }^{[16]}$, o gênero da pessoa de referência do domicílio (chefe da família) influi no nível de insegurança alimentar. Nos domicílios onde a pessoa de referência é uma mulher, a presença de insegurança alimentar é maior.

Os indicadores que compõem o IPVS da subprefeitura de Butantã (região em que se insere a população estudada), no ano 2000 , relatam que, nas regiões de muito alta vulnerabilidade social, pouco mais de $25 \%$ das famílias eram chefiadas por mulheres. Isto também foi verificado nas famílias estudadas, onde a mulher ainda é responsável, na maioria das vezes, pelos serviços domésticos e pelo cuidado das crianças.

Em situações onde o governo mantém-se omisso em relação a emprego, renda, moradia, meio físico, alimentação e saúde, a população desenvolve estratégias de enfrentamento para as dificuldades, como por exemplo, as redes de solidariedade familiar, comunitária ou institucional. Em populações submetidas à elevada vulnerabilidade social, a situação de privação na qual uma família encontra-se geralmente é amenizada pela mulher (pilar central do núcleo familiar, nestes casos), graças ao papel que ela desempenha na mobilização das redes familiares e comunitárias e pelo fato de que ela constitui, juntamente com as crianças, o núcleo central no que diz respeito à preparação e consumo de alimentos, os quais são oriundos da cozinha familiar, de doações de vizinhos e de amigos, de compras a fiado, da merenda escolar, da cesta básica e leite doados pela Pastoral da Criança ${ }^{[17]}$. 
Apesar da situação de insegurança alimentar ser prevista e esperada nas famílias estudadas, a sua constatação não se torna menos importante, uma vez que, em 1996, o Comitê de Direitos Econômicos, Sociais e Culturais das Nações Unidas, emitiu o Comentário Geral 12- Direito à Alimentação, que “ insiste na necessidade e obrigação que todo estado tem em respeitar, proteger e realizar o direito"[18].

O Brasil também tem colocado em sua agenda de discussão o tema da segurança alimentar e nutricional, estabelecendo em 2006, por meio da Lei Federal 11.346, a criação do sistema de segurança alimentar e nutricional (SISAN), para impulsionar políticas de Estado que garantam o direito à alimentação ${ }^{[19]}$.

Especial atenção deve ser dispensada à questão de que estas famílias apresentam-se compostas principalmente por crianças, com número representativo de gestantes e lactantes. Estes grupos possuem necessidades nutricionais aumentadas, tanto pelo crescimento e desenvolvimento fetal, quanto pela produção de leite. Apesar de receberem a doação de alimentos, 73 (88\%) famílias encontram-se em condição de insegurança alimentar, expostas ao risco nutricional.

Embora a presença de idosos nas famílias estudadas seja inexpressiva, verificou-se que os idosos não representam ônus para a família, uma vez que contribuem, de forma significativa, com a renda familiar, como no estudo de Marín-León et al ${ }^{[12]}$. Os idosos participantes da amostra eram aposentados ou pensionistas, contribuíam com a maior parte da renda familiar total e foram considerados pelos entrevistados como chefes da família.

Hoffmann ${ }^{[17]}$ atribui ao recebimento de aposentadorias ou pensões, pela estabilidade que apresenta, um efeito redutor da insegurança alimentar grave. Porém, somente o recebimento de aposentadoria ou pensão não é suficiente para alterar, de forma drástica, os níveis de insegurança alimentar, uma vez que fatores como renda familiar insuficiente, baixa escolaridade do chefe da família e elevado número de membros, que influem no grau de vulnerabilidade social e insegurança alimentar de uma população, estão muitas vezes presentes no grupo estudado.

Newmann et al ${ }^{[20]}$ indicam que a atuação da Pastoral da Criança, em locais de alta vulnerabilidade social e que necessitem de maiores investimentos por parte do governo, apresenta impacto positivo sobre os cuidados gerais e com a alimentação das crianças. A abordagem da Pastoral, que conta com três grandes momentos de intercâmbio de informações, representados pelas visitas domiciliares, dia da pesagem e reuniões mensais, poderia então minimizar a situação de insegurança alimentar familiar.

A habilitação das líderes da Pastoral da Criança "para iniciar a mobilização da comunidade a fim de enfrentar os problemas encontrados, tornando viável o caminho para a Segurança Alimentar e Nutricional no nível local", através da vigilância nutricional (pesagem mensal das crianças, orientação dos pais ou responsáveis quanto aos cuidados e o acompanhamento do peso e do crescimento da criança), incentivo à alimentação enriquecida e aproveitamento integral dos alimentos (ações que estimulam o aproveitamento de produtos de grande valor nutricional e de baixo custo, disponíveis nas próprias comunidades, além de evitar os desperdícios de grande parte dos alimentos, como folhas, cascas e sementes), provavelmente tem peso sobre os níveis de insegurança alimentar encontrados ${ }^{[21]}$. Há também o fato dos participantes locais da Pastoral da Criança serem integrantes do Centro de Referência em Segurança Alimentar e Nutricional do Butantã.

Ainda quanto à classificação das famílias em situação de segurança/insegurança alimentar, deve ser levado em conta que o instrumento utilizado - a EBIA - mede, com base nas respostas do entrevistado (geralmente mulher adulta responsável pela alimentação da casa), desde a preocupação e ansiedade das famílias no que diz respeito à possibilidade de acabar a comida antes de ser possível comprar mais, até a vivência de restrição alimentar grave, na qual adultos e crianças podem ficar um dia inteiro sem comer. Além disso, o instrumento "capta progressiva piora da situação, passando pelos niveis intermédios em que as famílias recorrem a estratégias de distribuição de menor quantidade de alimentos a seus membros, piora da qualidade nutritiva e diversificação da dieta, em função de reduziir custos, como ainda a supressão de algumas refeições, 
sendo as crianças poupadas nas situações de menor gravidade" [12].

Verificou-se que a entrevista com adoção do questionário da EBIA levava em média 15 minutos para ser realizada, fato que mostra sua viabilidade para a aplicação em larga escala, além do baixo custo para aplicação. Mesmo com tais vantagens, algumas dificuldades foram encontradas, as quais podem ser atribuídas ao entrevistador, ao ambiente da entrevista e ao entrevistado.

Com relação ao entrevistador, mostrou-se imprescindível que este desenvolvesse habilidade para conferir confiança ao entrevistado; enfatizar o período questionado (últimos 3 meses); apresentar tranqüilidade para lidar com possíveis momentos de demonstração de emoção pelo entrevistado; ressaltar as diferenças entre as diversas questões, que poderiam soar semelhantes se não fossem bem entendidas e mostrar que os problemas questionados referem-se somente à falta de condições para comprar ou receber alimentos. Por isso, a aplicação dos questionários de caracterização das famílias e de insegurança alimentar foi realizada por pesquisadores previamente treinados (alunos de graduação e pós-graduandos em Nutrição, aluno de graduação em Ciências Sociais e um Técnico em Nutrição).

O ambiente pode ser também desfavorável à aplicação do questionário da EBIA caso reúna condições que inibam o entrevistado, tais como: um local de entrevista que permita a circulação de terceiros, que apresente condições físicas insatisfatórias (local pouco arejado, demasiadamente pequeno e com assentos desconfortáveis) ou mesmo quando há a presença de acompanhantes do entrevistado. Levando-se em conta que o questionário foi aplicado em uma área de alta vulnerabilidade social, com estrutura física improvisada, circulação de terceiros e presença de acompanhantes, buscou-se realizar as entrevistas da forma mais discreta possível.

O entrevistado pode ser motivo de viés, devido à interpretação dos termos referentes à freqüência dos eventos ("quase todos os dias", "alguns dias" e "apenas um ou dois dias"), à quantidade de emagrecimento ("pouco", "médio" e "muito") e quanto à compreensão individual do termo "alimentação saudável e variada".

O MINISTÉRIO DE DESENVOLVIMENTO SOCIAL E COMBATE À FOME ${ }^{[2]]}$ destaca também que o entrevistado pode conferir vício de "prestígio" ou "benefício" à EBIA, caso as respostas dadas pelos entrevistados visem o recebimento de ajuda em alimentos ou benefícios sociais para seus domicílios e suas comunidades. Assim, foi fundamental que os indivíduos entrevistados soubessem que o objetivo do estudo não se encontrava atrelado a nenhum programa ou ação em Segurança Alimentar e Nutricional que envolvessem benefícios diretos ou indiretos.

Apesar da EBIA não capturar outras dimensões da segurança alimentar e nutricional, como contaminação microbiológica ou com metais pesados e pesticidas, e de ser limitada para o enfrentamento das condições que produzem a insegurança alimentar, centradas nos eixos da produção e acesso físico e econômico aos alimentos, não se pode deixar de destacar que o questionário da EBIA é um instrumento validado, o qual identifica a população de risco que deve ser alvo de programas públicos de caráter emergencial e situações relacionadas à fome no âmbito familiar e apresenta aplicação em toda população brasileira, seja ela rural ou urbana ${ }^{[3,22,23]}$.

\section{Conclusão}

A situação de insegurança alimentar encontrase prevalente nas famílias atendidas pela Pastoral da Criança no Jardim Jaqueline que participaram do estudo, as quais apresentam ainda renda familiar mensal insuficiente, baixa escolaridade, alto desemprego e más condições de habitação.

Como em outros estudos sobre a temática da segurança alimentar e nutricional, verificou-se que programas de transferência de renda, bem como investimentos na área da educação são imprescindíveis, uma vez que renda familiar e escolaridade são determinantes da insegurança alimentar.

A contribuição do presente trabalho 
manifesta-se pela identificação de algumas características sócio-demográficas e da situação de (in)segurança alimentar das famílias beneficiadas pelas ações da Pastoral da Criança, em uma região de alta vulnerabilidade social e por todas as informações indispensáveis para a elaboração, direcionamento e aperfeiçoamento de políticas públicas em segurança alimentar e nutricional.

Embora a EBIA apresente certas limitações quanto aos diferentes aspectos da segurança alimentar e nutricional, a escala é um instrumento validado nacionalmente, que pode ser utilizado em diferentes contextos sócio-econômicos e culturais. Assim, sugere-se, concomitantemente à aplicação da EBIA, a utilização de outros indicadores da situação de insegurança alimentar, como consumo alimentar domiciliar e disponibilidade de alimentos, os quais poderiam cooperar com os resultados obtidos pela escala, fornecendo também subsídios e indicativos para políticas públicas em segurança alimentar e nutricional.

\section{Referências bibliográficas}

[1] $2^{\circ}$ Conferência Nacional de Segurança Alimentar e Nutricional. Carta de Olinda. [documento na Internet]. Acesso em 18 ago 2005. Disponível em: http: //www.fomezero.gov.br/ download/ Carta $\% 20$ de $\% 20$ Olinda.pdf.

[2] Valente FLS. O direito humano à alimentação: desafios e conquistas. São Paulo: Cortez Editora, 2002. Do combate à fome à insegurança alimentar e nutricional: o direito à alimentação adequada; p. 3770.

[3] Sícoli JL. Pactuando conceitos fundamentais para a construção de um sistema de monitoramento da SAN. [documento na Internet]. Acesso em $30 \mathrm{mar}$ 2006. Disponível em: http: //www.polis.org.br/ download/65.pdf.

[4] Fundação SEADE. Espaços e Dimensões da Pobreza nos Municípios do Estado de São Paulo. [documento na Internet]. Acesso em 08 set 2005. Disponível em: http://www.seade.gov.br/produtos/ ipvs/analises/subprefeitura/butanta.pdf.
[5] Talher Nacional. Equipe de Educação Cidadã e Mobilização Social Um Brasil diferente está em nossas mãos. Brasília: Ministério de Desenvolvimento Social e Combate à Fome; 2004.

[6] SAS Secretaria Municipal de Assistência Social. Projeto caracterização de setores de alta vulnerabilidade social. São Paulo: Prefeitura da Cidade de São Paulo; 2003.

[7] Marques M, Costa C. Implementação de centros de referência local em segurança alimentar e nutricional. São Paulo: Instituto Pólis; 2005.

[8] Pastoral da Criança. Histórico, ações desenvolvidas, locais de atuação e organização. [site na Internet]. Acesso em 17 jan 2005. Disponível em: http:// www.pastoraldacrianca.org.br.

[9] Avelino ES, Pinheiro LTS, Fiore EG, Silva AM, David RM. Estado nutricional de crianças acompanhadas pela Pastoral da Criança em um bolsão de pobreza. In: Livro de resumos do 9으 Congresso Paulista de Saúde Pública, 2005 out 2226; Santos, BR. São Paulo: Saúde e Sociedade; 2005. p. 147.

[10] Segall-Correa AM. (In) Segurança alimentar no Brasil: Validação de metodologia para acompanhamento e avaliação de segurança alimentar de famílias brasileiras. Campinas; 2004. [relatório técnico]. Disponível em http://www.opas.org.br/ publicac.cfm.

[11] Pérez-Escamilla R, Segall-Correa AM, Maranha LK, Sampaio MFA, Marín-León L, Panigassi G. An adapted version of the U.S. Department of Agriculture Food Insecurity module is a valid tool for assessing household food insecurity in Campinas, Brazil. J Nutr. 2004; 134(8): 1923-8.

[12] Marín-León L, Segal-Corrêa AM, Panigassi G, Maranha LK, Sampaio MFA, Pérez-Escamilla R. A percepção de insegurança alimentar em famílias com idosos em Campinas, São Paulo, Brasil. Cad Saúde Pública. 2005; 21(5): 1433-40.

[13] Dean AG, Dean JA, Coulombier D. Epi Info ${ }^{\text {TM }}$ Version 6.04a, a word processing, database and 
statistics program for public health on IBMcompatible microcomputers. Atlanta: Centers for Disease Control and Prevention; 1996.

[14] Hoffmann R. Pobreza, insegurança alimentar e desnutrição no Brasil. Estudos Avançados. 1995; 9(24): 159-72.

[15] IBGE Instituto Brasileiro de Geografia e Estatística. Condições de Pobreza. [documento na Internet]. Acesso em 8 set 2005. Disponível em http:/ www.ibge. gov.br/ibgeteen/glossário/pobreza.html.

[16] Hoffman R. Determinantes da insegurança alimentar no Brasil. In: Anais do 14으 Congresso da Sociedade Latinoamericana de Nutrición; 2006; Florianópolis, BR.

[17] Gerhardt TE. Situações da vida, pobreza e saúde: estratégias alimentares e práticas sociais no meio urbano. Ciênc Saúde Coletiva. 2003; 8(3): 713-26.

[18] Belik W. Perspectivas para a segurança alimentar e nutricional no Brasil. Saúde Soc 2003; 12(1): 1220.

[19] Brasil. Lei no 11.346: criação do Sistema Nacional de Segurança Alimentar e Nutricional. Diário Oficial da União, 15 set 2006.

[20] Newmann NA, Victora CG, Halpern R, Guimarães PRV, César JA. Desempenho da Pastoral da Criança na promoção de ações de sobrevivência infantil e na educação em saúde em Criciúma, uma cidade do sul do Brasil. Pan Am J Public Health 1999; 5(6): 400-10.

[21] Conferência Nacional dos Bispos do Brasil.
Pastoral da Criança. Construindo caminhos para a segurança alimentar na comunidade: uma estratégia de planejamento participativo. Curitiba: Pastoral da Criança; 2000.

[22] MDS Ministério do Desenvolvimento Social e Combate à Fome. Indicadores de Insegurança Alimentar. [documento na Internet]. Acesso em 27 Abr 2006. Disponível em: http://www.mds.gov.br/ estatistica/Cadernos_2.pdf.

[23] Sampaio MFA, Kepple AW, Segall-Correa AM, Oliveira JTA, Panigassi G, Maranha LK et al. (In) Segurança Alimentar: experiência de grupos focais com populações rurais do Estado de São Paulo. Segurança Alimentar e Nutricional 2006; 13(1): 6477.

\section{Autores}

Daniela de Almeida Pereira - Aluna de Graduação da Faculdade de Saúde Pública da Universidade de São Paulo

Viviane Laudelino Vieira - Nutricionista do Centro de Saúde Escola Geraldo de Paula Souza da Faculdade de Saúde Pública da Universidade de São Paulo

Elaine Gomes Fiore - Professora Mestre da Universidade de Guarulhos

Ana Maria Cervato-Mancuso - Professora Doutora da Faculdade de Saúde Pública da Universidade de São Paulo Correspondência: Av. Dr. Arnaldo, 715 - Cerqueira César - CEP 01246-904 - São Paulo E-mail: cervato@usp.br 